\title{
Extracorporeal membrane oxygenation as a bridge to lung transplantation and recovery
}

\author{
Jeffrey Javidfar, MD, ${ }^{\mathrm{a}}$ Daniel Brodie, MD, ${ }^{\mathrm{b}}$ Alex Iribarne, MD, MS, ${ }^{\mathrm{a}}$ Julissa Jurado, MD, ${ }^{\mathrm{a}}$ \\ Matthew LaVelle, MSE, ${ }^{\mathrm{a}}$ Keith Brenner, MD, ${ }^{\mathrm{b}}$ Selim Arcasoy, MD, ${ }^{\mathrm{b}}$ Joshua Sonett, MD, ${ }^{\mathrm{a}}$ and \\ Matthew Bacchetta, MD ${ }^{\mathrm{a}}$
}

\begin{abstract}
Objective: Respiratory failure develops in many patients on lung transplant waiting lists before a suitable donor organ becomes available. Extracorporeal membrane oxygenation may be used to bridge such patients to recovery or lung transplantation.
\end{abstract}

\begin{abstract}
Methods: This is a review of a single-institution's experience with placing patients on extracorporeal membrane oxygenation with the intention of bridging them to lung transplantation. End points included successful bridging, duration of extracorporeal membrane oxygenation support, extubation, weaning from extracorporeal membrane oxygenation, overall survival, and extracorporeal membrane oxygenation-related complications. During an approximate 5-year period, acute respiratory failure developed in 18 patients (median age, 34 years) on the institution's lung transplant waiting list ( 8 hypoxemic, 9 hypercarbic, and 1 combined) who were placed on extracorporeal membrane oxygenation (13 venovenous and 5 venoarterial).
\end{abstract}

Results: All patients achieved appropriate extracorporeal membrane oxygenation blood flow rates (median, $4.05 \mathrm{~L} / \mathrm{min}$ ) and good gas exchange (median, on extracorporeal membrane oxygenation partial pressure of arterial carbon dioxide $43 \mathrm{~mm} \mathrm{Hg}$ and partial pressure of arterial oxygen $196 \mathrm{~mm} \mathrm{Hg}$ ). Thirteen patients (72\%) were successfully bridged: 10 to transplant and 3 returned to baseline function. Eleven patients $(61 \%)$ survived beyond 3 months, including the $10(56 \%)$ who underwent transplantation and are still alive. The median duration of extracorporeal membrane oxygenation support for patients who underwent transplantation was 6 days (3.5-31 days) versus 13.5 days (11-19 days) for those who did not undergo transplantation $(P=.45)$. Six patients $(33 \%)$ were extubated on extracorporeal membrane oxygenation, 4 of whom underwent transplantation. Four patients $(22 \%)$ who were too unstable for conventional interhospital transfer were transported on extracorporeal membrane oxygenation to Columbia University Medical Center. This subgroup had a $75 \%$ bridge to transplant or recovery rate and $100 \%$ survival in transplanted patients.

Conclusions: Extracorporeal membrane oxygenation is a safe and effective means of bridging well-selected patients with refractory respiratory failure to lung transplantation or return to their baseline condition. (J Thorac Cardiovasc Surg 2012;144:716-21)

Given the 12- to 24-month waiting period, patients with end-stage lung disease can acutely decompensate and develop refractory respiratory failure before suitable donor lungs become available. ${ }^{1}$ The need for prolonged invasive

From the Division of Cardiothoracic Surgery, ${ }^{\text {a }}$ Department of Surgery, Columbia University Medical Center, New York, NY; and Division of Pulmonary, Allergy, and Critical Care Medicine, ${ }^{b}$ Department of Medicine, Columbia University Medical Center, New York, NY.

Disclosures: Drs Bacchetta and Brodie previously did unpaid consulting for Maquet Cardiovascular, Inc. The remaining authors have nothing to disclose with regard to commercial support.

The authors had full control of the design of the study, methods used, outcomes, analysis of data, and production of the written report. The source of all funds to support this study and perform the evaluation was internal. There was no external source of funding for any phase of this study.

Received for publication Jan 18, 2012; revisions received April 22, 2012; accepted for publication May 16, 2012; available ahead of print July 16, 2012.

Address for reprints: Matthew Bacchetta, MD, 161 Fort Washington Ave, Herbert Irving Pavilion, Thoracic Surgery, Suite 301, New York, NY 10032 (E-mail: mb781 @ columbia.edu).

$0022-5223 / \$ 36.00$

Copyright (c) 2012 by The American Association for Thoracic Surgery

doi:10.1016/j.jtcvs.2012.05.040 mechanical ventilation may render these patients unsuitable for transplantation.

Patients with respiratory failure requiring mechanical ventilation can be temporarily supported on extracorporeal membrane oxygenation (ECMO) while they wait for a lung transplant if mechanical ventilation alone is insufficient to meet their gas exchange needs. ${ }^{2,3}$ ECMO may even allow some patients to be removed from mechanical ventilation while they await transplantation, which permits patients to eat, to participate in their own care, and to work more extensively with physical therapists, including ambulating with assistance. This has the potential to improve their pretransplant conditioning during this critical illness phase rather than allowing it to worsen, which is typically the case in these patients.

Venovenous ECMO is often sufficient to support such patients' physiologic needs. However, when there is significant pulmonary hypertension, an acute exacerbation can lead to right-sided heart failure that benefits from the ventricular unloading afforded by venoarterial ECMO. 


\section{Abbreviations and Acronyms \\ $\mathrm{ECMO}=$ extracorporeal membrane oxygenation \\ $\mathrm{ICU}=$ intensive care unit \\ $\mathrm{IQR}=$ interquartile range \\ $\mathrm{PaO}_{2}=$ partial pressure of arterial oxygen \\ $\mathrm{PaCO}_{2}=$ partial pressure of arterial carbon dioxide}

To date, the routine use of modern mechanical circulatory support to bridge patients to lung transplantation has been limited. ${ }^{4}$ The paucity of experience in the literature has prevented any durable conclusions regarding the safety and efficacy of such a strategy. Most previous reports have been limited by the use of multiple extracorporeal life support techniques, outdated technology, and small sample size. ${ }^{5,6}$

We report on the use of contemporary ECMO technology and management strategies to bridge patients listed for lung transplantation who subsequently developed an acute exacerbation of their underlying respiratory failure requiring invasive mechanical ventilation.

\section{PATIENTS AND METHODS}

This study, which was approved by the Columbia University Medical Center Institutional Review Board, is a retrospective review of a single institution's experience with bridging patients on the active lung transplant list to transplantation or recovery using ECMO support.

\section{Patient Selection}

The decision to place patients on ECMO was made by a team composed of thoracic surgeons, critical care intensivists, and transplant pulmonologists. To be a bridge candidate, a patient needed to be on the institution's active lung transplant waiting list. The indications for considering ECMO support were the presence of hypoxemic or hypercarbic respiratory failure requiring invasive mechanical ventilation with high levels of support or worsening right-sided heart failure. ${ }^{7}$ The lung diseases associated with hypoxemic respiratory failure included exacerbation of interstitial pulmonary fibrosis and pulmonary hypertensive crisis due to a congenital heart defect. In these patients, a partial pressure of arterial oxygen tension $\left(\mathrm{PaO}_{2}\right)$ to inspired oxygen fraction less than 80 was needed before initiation of ECMO. Hypercarbic respiratory failure (uncompensated hypercapnia with acidosis, $\mathrm{pH}<7.25$ despite optimal ventilator management) was seen in patients with an exacerbation of cystic fibrosis.

Patients who acquired a known contraindication to continuing ECMO support were decannulated. If a suitable donor lung was immediately available, patients were considered for transplant. Otherwise, the patient was delisted if temporarily maintaining the patient with mechanical ventilation alone was not feasible. Criteria for delisting a patient, whether temporarily or permanently, were consistent with institutional and United Network for Organ Sharing guidelines for all patients on the lung transplant waiting list. This included delisting patients who contracted and could not resolve Clostridium difficile colitis or who developed multiorgan system dysfunction while on ECMO. The institutional lung transplant selection committee made listing decisions. The transplant pulmonologists and cardiothoracic surgeons used the institution's standardized donor lung evaluation protocol to evaluate donor lungs for patients on ECMO.

\section{Protocol}

The cannulation techniques used were specific to the ECMO configuration deemed by the ECMO team to best serve the patient's physiologic needs. Patients were placed on venovenous ECMO via the internal jugular vein or venoarterial ECMO via femoral or subclavian arteries using previously described techniques. ${ }^{8,9}$ Patients with a congenital heart defect had the right internal jugular vein cannulated with a bicaval dual-lumen cannula under transesophageal echocardiographic guidance according to a previously described technique. ${ }^{10}$ The cannulae were attached to a standardized circuit. The ECMO circuit consisted of a Quadrox D or Quadrox I oxygenator (Maquet Inc, Rastatt, Germany) and a Jostra Rotaflow (Maquet Inc) or Levitronix Centrimag (Levitronix, GmbH, Zurich, Switzerland) centrifugal pump.

If patients on Columbia University Medical Center's lung transplant list decompensated at an outside hospital and were thought to be appropriate bridge to transplant candidates, they were cannulated at the outside hospital and transported via a mobile ECMO unit per standardized protocol. ${ }^{11}$ All patients were managed on ECMO according to a low-dose anticoagulation protocol (a partial thromboplastin time of 40-60 seconds). There was a high threshold for blood product transfusion with the goal of minimizing the creation of future antibodies, especially if a delay in transplantation was expected. $^{12,13}$ Leukocyte-depleted packed red blood cells were used for transfusion. Patients did not receive a transfusion unless it was needed to meet physiologic demand.

A review of the clinical records was conducted to obtain information regarding the patient's pre-ECMO status, operative course, hospitalization, and intensive care unit (ICU) stay. End points included successful bridging, duration of ECMO support, extubation, weaning from ECMO, overall survival, and ECMO-related complications.

\section{Statistical Methods}

All statistical analyses were performed with a statistical package (Stata 11; StataCorp LP, College Station, Tex). The total median ICU stay consisted of pre-ECMO, on-ECMO, and post-transplant ICU stays. Median values were provided with interquartile ranges (IQRs). To minimize potential concerns regarding normality of the data distribution, nonparametric rank-sum tests were used to compare continuous variables where appropriate. Categoric variables were compared using chi-square tests. The Wilcoxon Mann-Whitney rank-sum test, Fisher exact test, and univariate logistic regression were used for comparisons and subgroup analysis. A successful bridge was defined as any patient on the lung transplant waiting list who underwent transplantation or recovered after being placed on ECMO. Survival comparisons were made with Kaplan-Meier analysis, with survival estimates compared using a log-rank test. Overall survival was defined as the time from placement on ECMO to death or last follow-up through April 15, 2012. For the purpose of assessing statistical significance, a conventional alpha of 0.05 was used.

\section{Demographics}

From July 2007 to April 2012, 18 patients on the lung transplant list who experienced acute respiratory failure requiring invasive mechanical ventilation were placed on ECMO as a bridge to transplantation. This represents less than $10 \%$ of the total patients placed on ECMO during this period and is the entire institutional experience with bridging patients to lung transplantation using ECMO.

Nine patients experienced hypercarbic respiratory failure with a median pre-ECMO pH of 7.17 and median partial pressure of carbon dioxide of 123 $\mathrm{mm} \mathrm{Hg}$. One patient had combined hypercarbic and hypoxemic respiratory failure. The remaining 8 patients had hypoxemic respiratory failure with $\mathrm{PaO}_{2}$ to fraction of inspired oxygen of 63 (Table 1). The patients with pulmonary hypertensive crises had systemic or suprasystemic pulmonary artery pressures and right ventricular failure confirmed on transthoracic or transesophageal echocardiography.

The majority of patients ( $72 \%$ ) were supported for the entire extracorporeal life support course on venovenous ECMO. Five patients required venoarterial ECMO support. One patient was placed on femoral venoarterial 
TABLE 1. Study population characteristics

\begin{tabular}{|c|c|c|c|c|c|}
\hline \multirow{2}{*}{$\frac{\text { Variables* }}{\text { Demographics }}$} & \multicolumn{3}{|c|}{ Types of respiratory failure } & \multirow[b]{2}{*}{ Overall } & \multirow[b]{2}{*}{$P$ value } \\
\hline & Hypercarbic & Hypoxic & Combined & & \\
\hline Study population & $9(50 \%)$ & $8(44 \%)$ & $1(6 \%)$ & 18 & \\
\hline Age, y & $33(21-42)$ & $46(28-61)$ & 18 & $34(22-50)$ & .16 \\
\hline BMI & $23(19-25)$ & $22(17-27)$ & 16 & $22(19-25)$ & .67 \\
\hline Sex & & & & & .06 \\
\hline Male & 2 & 6 & 0 & 8 & \\
\hline Female & 7 & 2 & 1 & 10 & \\
\hline Pretransplant lung allocation score & $93(90-94)$ & $94(81-94)$ & 95 & $93(90-94)$ & .77 \\
\hline \multicolumn{6}{|l|}{ Reason for ECMO support } \\
\hline Cystic fibrosis exacerbation & 8 & & & 8 & \\
\hline Interstitial pulmonary fibrosis exacerbation & & 6 & & 6 & \\
\hline $\begin{array}{l}\text { Congenital atrial septal defect with pulmonary } \\
\text { hypertension and right ventricular failure }\end{array}$ & & 2 & & 2 & \\
\hline \multicolumn{6}{|l|}{ Respiratory variables } \\
\hline \multicolumn{6}{|l|}{ Pre-ECMO } \\
\hline $\mathrm{PaO}_{2} / \mathrm{FIO}_{2}$ & $200(74-228)$ & $63(58-71)$ & 63 & $74(63-200)$ & $<.01$ \\
\hline $\mathrm{pH}$ & $7.17(7.10-7.19)$ & $7.36(7.07-7.43)$ & 7.17 & $7.26(7.16-7.37)$ & .56 \\
\hline $\mathrm{PCO}_{2}, \mathrm{~mm} \mathrm{Hg}$ & $123(86-140)$ & $52(41-115)$ & 93 & $99(52-130)$ & .06 \\
\hline $\mathrm{PaO}_{2}, \mathrm{~mm} \mathrm{Hg}$ & $119(74-200)$ & $63(55-73)$ & 63 & $74(64-158)$ & $<.01$ \\
\hline $\mathrm{SaO}_{2}, \%$ & $96(92-97)$ & $89(78-92)$ & 88 & $92(89-97)$ & .02 \\
\hline \multicolumn{6}{|l|}{ On-ECMO } \\
\hline $\mathrm{pH}$ & $7.48(7.43-7.49)$ & $7.44(7.43-7.48)$ & 7.48 & $7.48(7.43-7.48)$ & .45 \\
\hline $\mathrm{PaCO}_{2}, \mathrm{~mm} \mathrm{Hg}$ & $47(43-56)$ & $40(32-43)$ & 55 & $43(40-49)$ & .01 \\
\hline $\mathrm{PaO}_{2}, \mathrm{~mm} \mathrm{Hg}$ & $241(137-335)$ & $196(108-348)$ & 109 & $196(109-335)$ & .96 \\
\hline $\mathrm{SaO}_{2}, \%$ & $100(99-100)$ & $99.7(98-100)$ & 99 & $100(99-100)$ & .45 \\
\hline \multicolumn{6}{|l|}{ Cannulation strategy } \\
\hline \multicolumn{6}{|l|}{ Venovenous } \\
\hline Single-site (internal jugular vein) & 7 & 4 & & 11 & - \\
\hline Dual-site (internal jugular and femoral vein) & & 2 & & 2 & - \\
\hline \multicolumn{6}{|l|}{ Venoarterial } \\
\hline Subclavian artery and internal jugular vein & 1 & 1 & & 2 & - \\
\hline Femoral artery and vein & 1 & & & 1 & - \\
\hline Femoral artery and internal jugular vein $\dagger$ & & 1 & 1 & 2 & - \\
\hline
\end{tabular}

BMI, Body mass index; ECMO, extracorporeal membrane oxygenation; $\mathrm{PaO}_{2}$, partial pressure of arterial oxygen; $\mathrm{FiO}_{2}$, fraction of inspired oxygen; $\mathrm{PaCO}$, partial pressure of arterial carbon dioxide; $\mathrm{SaO}_{2}$, arterial oxygen saturation. *Data are presented as number or median (IQR). †Conversion from venovenous to venoarterial ECMO.

ECMO because a venogram showed superior vena caval stenosis, which prohibited accommodation of a large venovenous ECMO catheter. Two patients had pulmonary hypertension with severe right-sided heart failure and were cannulated via the right subclavian artery. Two patients did not tolerate venovenous ECMO, as was evidenced by low cardiac output and persistent pressor requirements without improvement in end-organ oxygenation. These patients were transitioned to femoral venoarterial ECMO (Table 1).

\section{RESULTS}

The median duration of pre-ECMO mechanical ventilation was 18 hours (IQR, 12-24 hours). All patients underwent successful cannulation and were able to achieve appropriate blood flow rates for their catheter size (median, $4.05 \mathrm{~L} / \mathrm{min}$ ) and suitable gas exchange (on-ECMO partial pressure of carbon dioxide $43 \mathrm{~mm} \mathrm{Hg}$ and $\mathrm{PaO}_{2}$ $196 \mathrm{~mm} \mathrm{Hg}$ ). Four patients $(22 \%)$ were cannulated at an outside hospital and transported without any adverse events to Columbia University Medical Center while receiving ECMO.
The median duration of ECMO support was 11.5 days (IQR, 6-18 days). The median duration of ECMO support for those who underwent transplantation was 6 days (IQR, 4-18 days) versus 13.5 days (IQR, 11-19 days) for patients who did not undergo transplantation $(P=.45)$. One patient received double lung transplant after being bridged for 31 days on ECMO. Six patients $(33 \%)$ did not require a blood transfusion during their entire run on ECMO (Table 2). The total median ICU length of stay was 20 days (IQR, 16-32 days). The ICU stay was longer in the subgroup that underwent transplantation (22 vs 16.5 days for the group that did not undergo transplantation, $P=.35$ ). The median hospital length of stay was 36.5 days (22-51 days); however, it was shorter in the group that did not undergo transplantation ( 22.5 vs 47 days for the group that underwent transplantation) $(P=.07)$ (Table 2). This was attributable to the higher mortality in the group that did not undergo transplantation. 
TABLE 2. Extracorporeal membrane oxygenation performance parameters

\begin{tabular}{|c|c|c|c|c|}
\hline \multirow[b]{2}{*}{ Variable* } & \multicolumn{2}{|c|}{ Successful bridge } & \multirow[b]{2}{*}{ Failed bridge } & \multirow[b]{2}{*}{ Overall } \\
\hline & Transplantation & Returned to baseline function $\ddagger$ & & \\
\hline No. & 10 & 3 & 5 & 18 \\
\hline ECMO flow rate, $\mathrm{L} / \mathrm{min}$ & $4.05(3.3-4.5)$ & $3.9(3.25-4.3)$ & $4.20(3.5-4.4)$ & $4.05(3.7-4.4)$ \\
\hline Incidence of transfusion & 5 & 2 & 3 & $10(56 \%)$ \\
\hline pRBC transfused, units $\dagger$ & $0.16(0-0.17)$ & $0.27(0-0.30)$ & $0.16(0.07-0.26)$ & $0.16(0-0.25)$ \\
\hline Platelets transfused, $\mathrm{mL} \dagger$ & 0 & $0(0-28)$ & $0(0-5)$ & 0 \\
\hline Duration of ECMO, d & $6(3.5-18)$ & $11(2-23)$ & $15(12-17)$ & $11.5(6-18)$ \\
\hline ICU length of stay, $d$ & $22(18-33)$ & $16(14-94)$ & $17(16-23)$ & $20(16-32)$ \\
\hline Hospital length of stay, $d$ & $47(41-52)$ & $22(16-178)$ & $23(16-28)$ & $36.5(22-51)$ \\
\hline
\end{tabular}

Thirteen patients $(72 \%)$ were eventually liberated from mechanical ventilation after recovering to their baseline or being successfully bridged to transplant. Ten patients $(56 \%)$ underwent transplantation (8 double and 2 single lung transplants), and all are alive to date. This represents $4 \%$ of the approximately 250 total lung transplants performed during the duration of the study period at Columbia University Medical Center. Severe primary graft dysfunction developed in 2 patients who underwent transplantation, and they required a second course of venovenous ECMO. All patients currently have a viable, functioning graft (Table 3 ).

Eight patients (44\%) died, none of whom received transplants. This includes the 3 patients who transiently recovered their baseline function and were extubated after being weaned from ECMO. One patient survived beyond 3 months. Two patients were delisted after decannulation and were extubated. One patient survived 2 weeks, and 1 patient survived 2 months. The causes of mortality were multisystem organ failure $(n=6)$, pneumonia $(n=1)$, and sudden cardiac arrest $(\mathrm{n}=1)$. ECMO-related complications included a deep venous thrombosis that resolved with anticoagulation after transplantation and suspected heparininduced thrombocytopenia requiring Argatroban (GlaxoSmithKline, Middlesex, UK) (Table 3).

\section{DISCUSSION}

This series examines a high-volume ECMO center's experience with using peripheral extracorporeal support as a means of bridging patients to lung transplant or recovery after an episode of acute respiratory failure. This experience reinforces other studies performed at centers in the United States and Europe., ${ }^{214}$ Other means of bridging patients with end-stage lung disease, including the Novalung (Novalung GmbH, Heilbronn, Germany) interventional lung assist device or percutaneous extracorporeal carbon dioxide exchange, have notable disadvantages, such as femoral access and inability to provide complete respiratory support, respectively. ${ }^{15,16}$ Accordingly, centers have moved away from these technologies when bridging patients to lung transplant.
Venovenous ECMO is our center's preferred approach for bridging patients to lung transplantation because it avoids the potential complications associated with the arterial cannula, such as limb ischemia, and can often be accomplished through a single peripheral cannula. However, 1 patient with

TABLE 3. Study population outcomes

\begin{tabular}{|c|c|c|}
\hline Variable & No. & Range or $\%$ \\
\hline ECMO results & 18 & 100 \\
\hline Mechanical ventilator pre-ECMO, $\mathrm{h}$ & 18 & $(12-24)^{*}$ \\
\hline Duration of ECMO support, d & 11.5 & $(6-18)$ \\
\hline Successful wean from ECMO/decannulated & 17 & 94 \\
\hline Liberation from mechanical ventilation & 13 & 72 \\
\hline $\begin{array}{l}\text { Liberation from mechanical ventilation while on } \\
\text { ECMO }\end{array}$ & 6 & 33 \\
\hline Successful bridge $\dagger$ & 13 & 72 \\
\hline Bridge to transplant & 10 & 56 \\
\hline Bridge to recovery/baseline function & 3 & 17 \\
\hline Ambulation on ECMO & 5 & 28 \\
\hline Bike riding on ECMO & 2 & 11 \\
\hline \multicolumn{3}{|l|}{ Survival and follow-up } \\
\hline Died while on ECMO & 1 & 6 \\
\hline Died after decannulation from ECMO & 5 & 28 \\
\hline Discharged from ICU & 13 & 72 \\
\hline Discharged from hospital & 11 & 61 \\
\hline Severe primary graft failure $(\mathrm{N}=10$ transplanted) & 2 & $20 \ddagger$ \\
\hline \multicolumn{3}{|l|}{ Survival } \\
\hline $1 \mathrm{mo}$ & 12 & 67 \\
\hline \multicolumn{3}{|l|}{ Bridged to transplant $\S$} \\
\hline $1 \mathrm{mo}(\mathrm{N}=10$, at risk $) \S$ & 10 & 100 \\
\hline $6 \mathrm{mo}(\mathrm{N}=7$, at risk $)$ & 7 & 100 \\
\hline $1 \mathrm{y}(\mathrm{N}=6$, at risk $)$ & 6 & 100 \\
\hline $2 \mathrm{y}(\mathrm{N}=3$, at risk $)$ & 3 & 100 \\
\hline $\begin{array}{l}\text { Overall survival in subgroup that underwent } \\
\text { transplantation }\end{array}$ & 10 & 100 \\
\hline \multicolumn{3}{|l|}{ ECMO-related complications } \\
\hline Heparin-induced thrombocytopenia & 1 & 6 \\
\hline Deep venous thrombosis at cannulation site & 1 & 6 \\
\hline Conversion to venoarterial ECMO & 2 & 11 \\
\hline
\end{tabular}

$E C M O$, Extracorporeal membrane oxygenation; $I C U$, intensive care unit. *Interquartile range. $\dagger$ Received transplant or recovered to baseline function. $\ddagger$ Of subgroup. $\S$ Bridged to transplant only. 

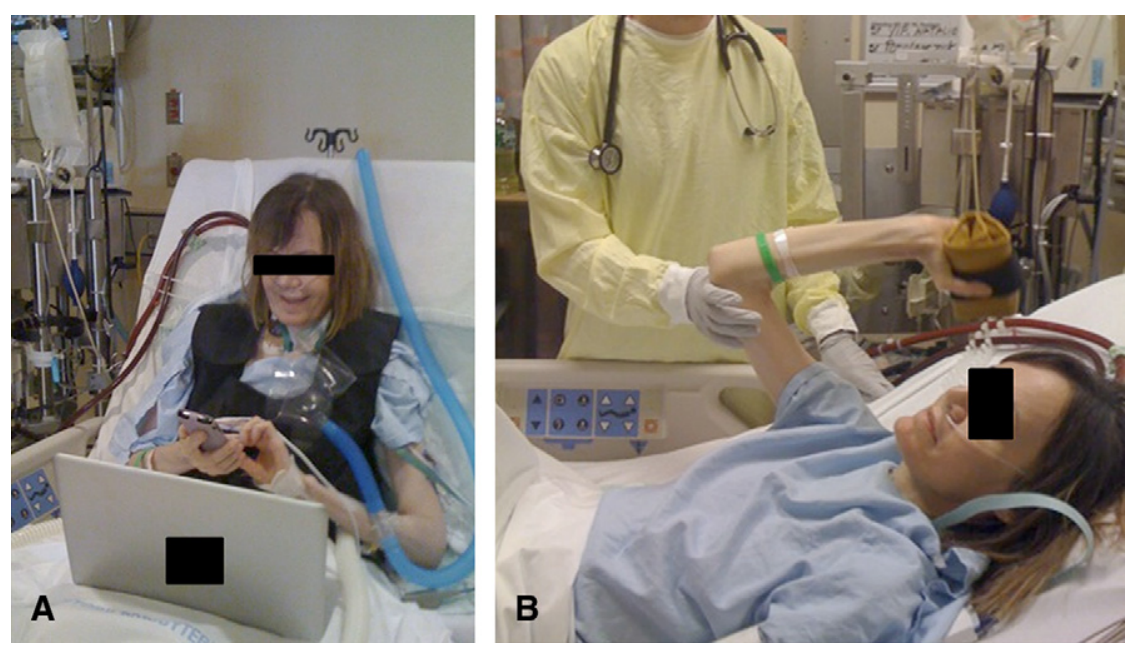

FIGURE 1. The 6 patients who were extubated while on ECMO participated in physical therapy and activities of daily living before undergoing transplantation. This patient on ECMO is (A) using her cellular telephone and laptop computer and (B) performing arm-strengthening exercises with the physical therapist.

primary respiratory failure was inadequately supported with venovenous ECMO and was converted to peripheral venoarterial ECMO.

Upper-body ECMO cannulation (via the internal jugular vein and subclavian artery-when venoarterial ECMO is needed) facilitates patient mobilization and participation in physical therapy. ${ }^{13}$ Six patients were extubated while on ECMO. Five patients ambulated around the ICU, and 2 patients rode an upright stationary bike. During ambulation, patients used a rolling walker while a streamlined ECMO circuit (pump, oxygenator, and oxygen source) on a roller chart followed in tow. Early patient mobilization while on ECMO minimizes physical deconditioning, which allows the patient to better tolerate lung transplantation (Figure 1).

In addition to early mobilization, patients are able to eat and drink normally and participate in ongoing counseling, which helps them better handle the psychologic rigors and stresses of both the bridge period and the immediate posttransplant phase. ${ }^{17}$ The combined benefits of extubating and ambulating patients on ECMO may offset any potential drawbacks of a prolonged bridge period. These steps may actually improve patients' suitability for lung transplant during the course of their critical illness. Likewise, a conscious effort to minimize transfusions during the ECMO period may reduce the risk of antibody generation, although such an effect is likely small given the time frame required to develop antibodies relative to the waiting time for donor lungs.

The combination of transporting patients on ECMO and bridging them to lung transplantation was found to be a safe and effective means of bringing patients to an experienced transplant center. This is especially important because lung transplantation is typically limited to major medical centers that are often located in urban areas. Therefore,
ECMO technology can expand treatment options for patients who live in remote areas. This concept has been shown to be feasible in select centers in Europe. ${ }^{2}$

The cause of respiratory failure did not affect rates of decannulation, extubation, transplantation, or overall survival. The duration of pre-ECMO mechanical ventilator support was brief, but this can be attributed to the fact that the patients rapidly decompensated, as seen in the pre-ECMO arterial blood gas, and did not tolerate maximal conventional respiratory support.

Patients tolerated prolonged periods of time (up to 1 month) on ECMO and were still deemed suitable transplant candidates. $^{4,18}$ However, bridging a patient on ECMO is not an indefinite process. Once patients were deemed to no longer be transplant candidates, they were delisted and weaned from ECMO. Strict patient selection in terms of transplantation may have contributed to superior early results in the population who underwent transplantation. ${ }^{4}$

Patients who underwent transplantation were all discharged from the hospital and have a $100 \%$ survival through July 1, 2012 (Figure 2). In this exploratory analysis, the survival figures for the population of lung transplant recipients bridged on ECMO are not inferior to other transplant recipients given the median lung allocation score of 93. Comparatively, on the basis of an analysis of United Network for Organ Sharing data, lung transplant recipients with a lung allocation score greater than 90 have a $50 \%$ survival at 2 years. ${ }^{19}$ Longer follow-up and more patients are needed to validate our preliminary clinical experience.

This study was further limited by its single-institution, retrospective nature. Although attempting to bridge 18 patients to transplantation over an approximately 5-year period was a relatively substantial experience, the study was inadequately powered to discern significant differences at 


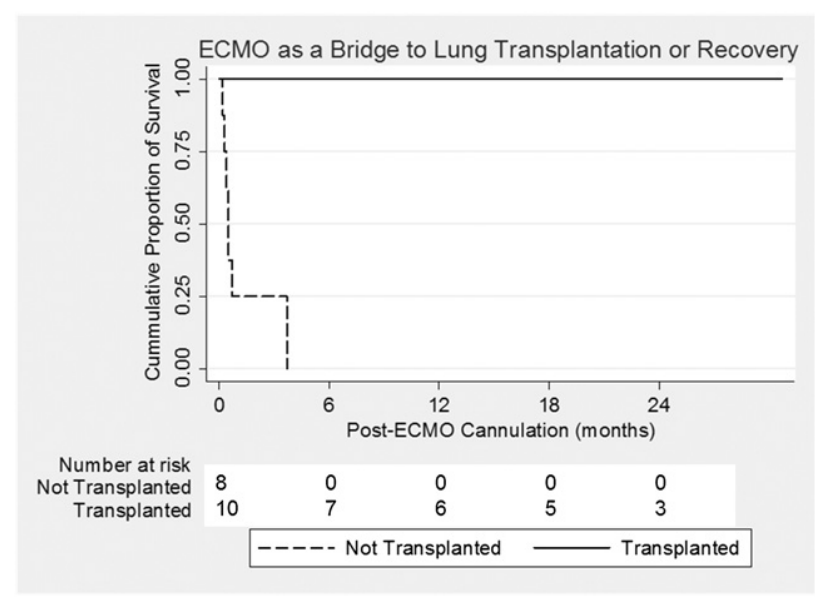

FIGURE 2. Survival in patients bridged on ECMO to lung transplantation or recovery. ECMO, Extracorporeal membrane oxygenation.

the subgroup level. A multicenter, prospective randomized study would likely elucidate these differences.

\section{CONCLUSIONS}

ECMO is a feasible, safe, and effective means of bridging patients to transplantation who develop an exacerbation of their end-stage lung disease resulting in acute respiratory failure. ECMO offers promise to patients who might otherwise die before suitable donor lungs become available.

\section{References}

1. Scientific Registry of Transplant Recipients. Available at: http://www.srtr.org/. Accessed December 29, 2011.

2. Haneya A, Philipp A, Mueller T, et al. Extracorporeal circulatory systems as a bridge to lung transplantation at remote transplant centers. Ann Thorac Surg. 2011;91:250-5.

3. Hämmäinen $P$, Schersten $H$, Lemström $K$, et al. Usefulness of extracorporeal membrane oxygenation as a bridge to lung transplantation: a descriptive study. J Heart Lung Transplant. 2011;30:103-7.
4. Bermudez CA, Rocha RV, Zaldonis D, et al. Extracorporeal membrane oxygenation as a bridge to lung transplant: midterm outcomes. Ann Thorac Surg. 2011; 92:1226-32.

5. Aigner C, Wisser W, Taghavi S, et al. Institutional experience with extracorporeal membrane oxygenation in lung transplantation. Eur J Cardiothorac Surg. 2007;31:468-74

6. Jackson A, Cropper J, Pye R, Junius F, Malouf M, Glanville A. Use of extracorporeal membrane oxygenation as a bridge to primary lung transplant: 3 consecutive, successful cases and a review of the literature. J Heart Lung Transplant. 2008;27:348-52.

7. Javidfar J, Brodie D, Wang D, et al. Use of bicaval dual-lumen catheter for adult venovenous extracorporeal membrane oxygenation. Ann Thorac Surg. 2011;91: 1763-9.

8. Javidfar J, Wang D, Zwischenberger JB, et al. Insertion of bicaval dual lumen extracorporeal membrane oxygenation catheter with image guidance. ASAIO J. 2011;57:203-5.

9. Javidfar J, Brodie D, Costa J, et al. Subclavian artery cannulation for venoarterial extracorporeal membrane oxygenation. ASAIO J. 2012 (in press).

10. Javidfar J, Brodie D, Sonett J, Bacchetta M. Venovenous extracorporeal membrane oxygenation using a single cannula in patients with pulmonary hypertension and atrial septal defects. J Thoracic Cardiovasc Surg. 2012 143:982-4.

11. Javidfar J, Brodie D, Takayama H, et al. Safe transport of critically ill adult patients on extracorporeal membrane oxygenation support to a regional extracorporeal membrane oxygenation center. ASAIO J. 2011;57:421-5.

12. Combes A, Bacchetta M, Brodie D, Muller T, Pellegrino V. Extracorporeal membrane oxygenation for respiratory failure in adults. Curr Opin Crit Care. 2012; 18:99-104.

13. Brodie D, Bacchetta M. Extracorporeal membrane oxygenation for ARDS in adults. $N$ Engl J Med. 2011;365:1905-14.

14. Nosotti M, Rosso L, Palleschi A, et al. Bridge to lung transplantation by venovenous extracorporeal membrane oxygenation: a lesson learned on the first four cases. Transplant Proc. 2010;42:1259-61.

15. Fischer S, Simon AR, Welte T, et al. Bridge to lung transplantation with the nove pumpless interventional lung assist device NovaLung. J Thorac Cardiovasc Surg. 2006;131:719-23.

16. Zwischenberger JB, Conrad SA, Alpard SK, Grier LR, Bidani A. Percutaneous extracorporeal arteriovenous $\mathrm{CO} 2$ removal for severe respiratory failure. Ann Thorac Surg. 1999;68:181-7.

17. Olsson KM, Simon A, Strueber M, et al. Extracorporeal membrane oxygenation in nonintubated patients as bridge to lung transplantation. Am J Transplant. 2010; 10:2173-8.

18. Broome M, Palmer K, Schersten H, Frenchner B, Nilsson F. Prolonged extracorporeal membrane oxygenation and circulatory support as bridge to lung transplant. Ann Thorac Surg. 2008;86:1357-60.

19. Russo MJ, Worku B, Iribarne A, et al. Does lung allocation score maximize surviva benefit from lung transplantation? J Thorac Cardiovasc Surg. 2011;141:1270-7. 